Pacific Journal of Mathematics

AN ANALOGUE OF THE PALEY-WIENER THEOREM FOR 


\title{
AN ANALOGUE OF THE PALEY-WIENER THEOREM FOR CERTAIN FUNCTION SPACES ON $S L(2, \boldsymbol{C})$
}

\author{
ANDREW B. WANG
}

The classical theorem of Paley-Wiener is concerned with characterizing Fourier transforms of $C^{\infty}$ functions of compact support on the real line. It states that an entire holomorphic function $F$ is the Fourier-Laplace transform of a $C^{\infty}$ function on the real line $R$ with support in $|x| \leqq R$ it and only if for given integer $m$, there exists a constant $C_{m}$ such that

$$
|F(\xi+i \eta)| \leqq C_{m}(1+|\xi+i \eta|)^{-m} \exp R|\eta|, \quad \xi, \eta \in \boldsymbol{R} .
$$

The purpose of this paper is to prove an analogue of this theorem for certain convolution subalgebras of $C^{\infty}$ functions with compact support on the group $S L(2, \boldsymbol{C})$, by using Fourier transform involving elementary spherical functions of general type $\delta$.

These subalgebras have been defined on locally compact group by $R$. Godement [4], in order to study the spherical trace function, cf. also G. Warner [8]. On this special group mentioned, by use the differential equations satisfied by the spherical functions, we derive a parametrization of such functions. These are in turn utilized to prove the Paley-Wiener theorem.

The analogous question on symmetric space of noncompact type was considered by S. Helgason [5] and R. Gangolli [3]. L. Ehrenpreis and F. I. Mautner [2] studied the Fourier transform on the group $S L(2, R)$ in detail, and theorem of the same kind was proved there. Results of this sort involving spherical functions of general type $\delta$ on some other groups have also been investigated, see e.g. Y. Shimizu [7].

2. Preliminaries. Throughout this paper, let $G$ denote the complex semisimple Lie group $S L(2, C)$ and let $K$ denote the maximal compact subgroup consisting of all unitary matrices in $G$. A basis of the real Lie algebra $g_{0}$ of $G$ consists of

$$
\begin{array}{ll}
R_{1}=\frac{1}{2}\left(\begin{array}{rr}
0 & 1 \\
-1 & 0
\end{array}\right), \quad R_{2}=\frac{1}{2}\left(\begin{array}{ll}
0 & i \\
i & 0
\end{array}\right), \quad R_{3}=\frac{1}{2}\left(\begin{array}{rr}
i & 0 \\
0 & -i
\end{array}\right) \\
S_{1}=\frac{1}{2}\left(\begin{array}{rr}
0 & -i \\
i & 0
\end{array}\right), \quad S_{2}=\frac{1}{2}\left(\begin{array}{ll}
0 & 1 \\
1 & 0
\end{array}\right), \quad S_{3}=\frac{1}{2}\left(\begin{array}{rr}
1 & 0 \\
0 & -1
\end{array}\right) .
\end{array}
$$

The set $\left\{R_{1}, R_{2}, R_{3}\right\}$ also forms a basis of the Lie algebra $k_{0}$ of $K$. 
Elements of $g_{0}$ are viewed as left invariant vector fields on $G$, which generates the algebra $\&$ of all left invariant differential operators on $G$. Let $a_{p_{0}}=\left\{t S_{3}: t \in \boldsymbol{R}\right\}$. The root system for $\left(g_{0}, a_{p_{0}}\right)$ consists of $\{\rho,-\rho\}$, where $\rho\left(S_{3}\right)=1$, and each has multiplicity two. Let $N_{1}=\left(\begin{array}{ll}0 & 1 \\ 0 & 0\end{array}\right), N_{2}=\left(\begin{array}{ll}0 & i \\ 0 & 0\end{array}\right)$ and let $\mathfrak{n}_{0}$ be the subspace of $g_{0}$ spanned by $\left\{N_{1}, N_{2}\right\}$, then $\mathfrak{n}_{0}$ is the root space for $\rho$. Let $N=\exp \mathfrak{n}_{0}$ and $A_{p}=\left\{a_{t}=\exp t S_{3}: t \in \boldsymbol{R}\right\}$. Then $g_{0}=k_{0}+a_{p_{0}}+\mathfrak{n}_{0}$ and $G=K A_{p} N$ (Iwasawa decomposition). It is also known that $G=K A_{p}^{+} K, A_{p}^{+}=$ $\left\{a_{t}: t \geqq 0\right\}$. The Haar measure on $G$ is normalized so that

$$
\int_{G} f(x) d x=\int_{K} \int_{A_{p}} \int_{N} f\left(k a_{t} n\right) e^{2 t} d k d t d n, \quad f \in C_{c}(G),
$$

where $d k$ is the normalized Haar measure on $K, d t$ is the Lebesgue measure on $R$ and $d n=d \xi_{1} d \xi_{2}$ if $n=\exp \left(\xi_{1} N_{1}+\xi_{2} N_{2}\right)$, is the Lebesgue measure on $R^{2}$. Let $k \in K$, we can write $k=u_{\varphi_{1}} v_{\theta} u_{\varphi_{2}}$ with $u_{\varphi}=$ $\exp \varphi R_{3}, v_{\theta}=\exp \theta R$, and $0 \leqq \varphi_{1} \leqq 2 \pi, 0 \leqq \varphi_{2} \leqq 4 \pi$. Then

$$
\int_{K} f(k) d k=\frac{1}{16 \pi^{2}} \int_{\varphi_{1}=0}^{2 \pi} \int_{\theta=0}^{\pi} \int_{\varphi_{2}=0}^{4 \pi} f\left(u_{\varphi_{1}} v_{\theta} u_{\varphi_{2}}\right) \sin \theta d \varphi_{1} d \theta d \varphi_{2},
$$

$$
f \in C(K) \text {. }
$$

For each nonnegative integer or half integer $s$, let $D^{s}$ be the unique (up to equivalence) irreducible unitary representation of $K$ on a $2 s+1$ dimensional Hilbert space $E_{s}$. We can choose a basis $\left\{v_{-s}\right.$, $\left.v_{-s+1}, \cdots, v_{s}\right\}$ of $E_{s}$ so that the matrix $\left(D_{j, q}^{s}(k)\right), j, q=-s,-s+1$, $\cdots, s$ has the following expression [see e.g. 6, p. 129].

$$
\begin{aligned}
D_{j, q}^{s}\left(u_{\varphi}\right)= & \delta_{j, q} e^{-i q \varphi} \\
D_{j, q}^{s}\left(v_{\theta}\right)= & (-1)^{j-q}\left(\frac{(s+j) !(s-j) !}{(s+q) !(s-q) !}\right)^{1 / 2} \\
& \times \sum_{r=\max \{0, q-j !}^{\min \{s-j, s+q\}}(-1)^{r}\left(\begin{array}{c}
s+q \\
r
\end{array}\right)\left(\begin{array}{c}
s-q \\
s-j-r
\end{array}\right) \\
& \cos ^{2 s-j+q-2 s} \frac{\theta}{2} \sin ^{j-q+2 r} \frac{\theta}{2} .
\end{aligned}
$$

The infinitesimal form for $D^{s}$ has

$$
\begin{aligned}
& D^{s}\left(R_{1}\right) v_{j}=\frac{1}{2}(s+j) v_{j-1}-\frac{1}{2}(s-j) v_{j+1} \\
& D^{s}\left(R_{2}\right) v_{j}=\frac{1}{2}(s+j) v_{j-1}+\frac{1}{2}(s-j) v_{j+1} \\
& D^{s}\left(R_{3}\right) v_{j}=-i j v_{j} .
\end{aligned}
$$

Hence $D^{s}\left(R_{1}^{2}+R_{2}^{2}+R_{3}^{2}\right)=-s(s+1) I$. 
Let $M=\left\{u_{\theta}=\exp \theta S_{3}: \theta \in R\right\}$. Then $M$ is the centralizer of $A_{p}$ in $K$, also it is a maximal torus in $K$. The set $\hat{M}$ of all characters of $M$ is parametrized by half integers, i.e., for each $p$ with $2 p$ an integer, $u_{\theta} \rightarrow e^{-i p \theta}$ gives a character of $M$. Let $p \in \hat{M}$, and let $E^{p}=\left\{f \in L^{2}(K): f\left(k u_{\theta}\right)=e^{i \rho \theta} f(k), k \in K\right.$ and $\left.u_{\theta} \in M\right\}$, with $\left\|f^{2}\right\|=$ $\int_{K}|f(k)|^{2} d k$. Let $\lambda$ be a complex number and given $x \in G$, define $U^{p, \lambda}(x)$ by the prescription

$$
\left(U^{p, \lambda}(x) f\right)(k)=\exp \left(-(i \lambda+1) \rho\left(H\left(x^{-1} k\right)\right) f\left(k\left(x^{-1} k\right)\right), \quad f \in E^{p}\right.
$$

where $x=\kappa(x) \cdot \exp H(x) \cdot n(x)$ is the Iwasawa decomposition for $x$. Then $U^{p, \lambda}$ defines a continuous representation of $G$ on the Banach space $E^{p}$, and every TCI Banach representation of $G$ is equivalent to a subquotient of $U^{p, \lambda}$ for some $p, \lambda$. The restriction of $U^{p, \lambda}$ to $K$ is just the unitary representation of $K$ induced from the character $u_{\theta} \rightarrow e^{i p \theta}$ of $M$, hence $D^{s}$ occurs in $U^{p, \lambda}$ exactly once if and only if $s=|p|+q$ for some nonnegative integer $q$.

$U^{p, \lambda}$ is unitary if $\lambda$ is real, which constitutes the principal series representation induced from the characters of the group $M A_{p} N$. Define

$$
U^{p, \lambda}(f)=\int_{G} f(x) U^{p, \lambda}(x) d x, \quad f \in C_{c}^{\infty}(G) .
$$

Then $U^{p, \lambda}(f)$ is of trace class and we have the inversion formula

$$
f(x)=\frac{1}{4 \pi^{3}} \sum_{2 p \in z} \int_{\lambda=-\infty}^{\infty}\left(p^{2}+\lambda^{2}\right) \operatorname{Trace}\left(U^{p, \lambda}\left(x^{-1}\right) U^{p, \lambda}(f)\right) d \lambda
$$

where $Z$ is the set of all integers and $d \lambda$ is the usual Euclidean measure.

3. The spherical functions. Let $C_{c}^{\infty}(G)$ be the algebra of all $C^{\infty}$ functions with compact support on $G$, with multiplication defined by convolution. The subalgebra $I_{c}(G)$ is formed by those functions $f$ in $C_{c}^{\infty}(G)$ satisfying $f\left(k x k^{-1}\right)=f(x)$ for $x \in G, k \in K$. Define $\chi_{s}(k)=$ $(2 k+1)$ Trace $\left(D^{s}(k)\right), k \in K$ and $D^{s} \in \hat{K}$. Let $C_{c, s}(G)=\left\{f \in C_{c}^{\infty}(G): f * \chi_{x}=\right.$ $\left.f=\chi_{s}^{*} f\right\}$ and $I_{c, s}(G)=I_{c}(G) \cap C_{c, s}(G) . \quad I_{c, s}(G)$ is a subalgebra of $C_{c}^{\infty}(G)$ and the mapping $f \rightarrow f^{0 *} \chi_{s}, f^{0}(x)=\int_{K} f\left(k x k^{-1}\right) d k$, is the projection of $C_{c}^{\infty}(G)$ onto $I_{c, s}(G)$.

Definition. Let $D^{s} \in \hat{K}$. By a spherical function $\Phi$ on $G$ of type $s$ we mean a quasi-bounded continuous function on $G$ such that (i) $\Phi\left(k x k^{-1}\right)=\Phi(x), x \in G$ and $k \in K$; (ii) $\Phi^{*} \chi_{s}=\Phi$; (iii) the map $f \rightarrow$ $\int_{G} f(x) \Phi(x) d x$ is a nonzero homomorphism of the algebra $I_{c, s}(G)$ onto 
the complex numbers $C$.

Spherical functions of type $s$ relates naturally to the TCI Banach representations of $G$. Suppose $U$ is a TCI Banach representation of $G$ on a space $E$ such that $D^{s}$ occurs in the restriction of $U$ to $K$. Let $U\left(\chi_{s}\right)=\int_{K} U(k) \chi_{s}(k) d k$ and $E(s)=U\left(\chi_{s}\right) E$. The $s$-spherical function $\Psi_{s}^{U}$ of $U$ on $G$ is defined by $\Psi_{s}^{U}(x)=U\left(\chi_{s}\right) U(x) U\left(\chi_{s}\right)$. Since $D^{s}$ occurs in $U$ exactly once, choose a basis for $E(s)$ so that $U(k)=D^{s}(k)$ on $E(s)$. Then clearly $\Psi_{s}^{U}\left(k_{1} x k_{2}\right)=D^{s}\left(k_{1}\right) \Psi_{s}^{U}(x) D^{s}\left(k_{2}\right)$. Let $\Psi_{s, K}^{U}(x)=$ $\int_{K} \Psi_{s}^{U}\left(k x k^{-1}\right) d k$. Then $\Psi_{s, K}^{U}(x) D^{s}(k)=D^{s}(k) \Psi_{s}^{U}(x), x \in G, k \in K$, and we have $\Psi_{s, K}^{U}(x)$ is a scalar $\Phi_{s}^{U}(x)$ times identity operator. We recall the following facts, [cf. 8, Ch. 6].

Proposition 3.1. (i) $\Phi_{s}^{U}$ is a spherical function of type $s$ and every spherical function of type $s$ is of this form.

(ii) Let $\kappa_{U}$ be the infinitesimal character of $U$ defined on the center 3 of the algebra $\mathbb{S}$, then $D \Phi_{s}^{U}=\kappa_{U}(D) \Phi_{s}^{U}$ and $D \Psi_{s}^{U}=\kappa_{U}(D) \Psi_{s}^{U}$, $D \in 3$.

Consider the Banach representation $U^{p, \lambda}$ with $s=|p|+q$ for some nonnegative integer $q$, let $\Psi_{s}^{p, \lambda}$ and $\Phi_{s}^{p, \lambda}$ be the $s$-spherical function and the spherical function of type $s$ respectively of the TCI Banach representation of $G$ which occurs in $U^{p, \lambda}$ and has $D^{s}$ occurs in it. Let $E^{p}(s)=U^{p, \lambda}\left(\chi_{s}\right) E^{p}$, then $\left\{D_{j,-p}^{s}: j=-s,-s+1, \cdots, s\right\}$ forms a basis for $E^{p}(s)$. Now

$$
\begin{aligned}
\Psi_{s}^{p, \lambda}(x) \cdot D_{j,-p}^{s}= & U^{p \lambda}\left(\chi_{s}\right) U^{p, \lambda}(x) U^{p, \lambda}\left(\chi_{s}\right) D_{j,-p}^{s} \\
= & (2 s+1) \sum_{l=-s}^{s} \int_{K} \exp \left(-(i \lambda+1) \rho\left(H\left(x^{-1} k\right)\right)\right) \\
& \times D_{j,-p}^{s}\left(\kappa\left(x^{-1} k\right)\right) d k \cdot D_{l,-p}^{s} .
\end{aligned}
$$

But $\Phi_{s}^{p, \lambda}(x)=1 /(2 s+1)$ Trace $\left(\Psi_{s, K}^{p, \lambda}(x)\right)=1 /(2 s+1)$ Trace $\left(\Psi_{s}^{p, \lambda}(x)\right)$, so

$$
\Phi_{s}^{p, \lambda}(x)=\int_{K} \exp \left(-(i \lambda+1) \rho\left(H\left(x^{-1} k\right)\right) D_{-p,-p}^{s}\left(k^{-1} \kappa\left(x^{-1} k\right)\right) d k .\right.
$$

Using this formula and the above proposition, we will set up a differential equation which enables us to get a complete parametrization of the spherical functions of type $s$.

LeMma 3.2. $\Phi_{s}^{p, \lambda}(x)=\Phi_{s}^{-p,-\lambda}\left(x^{-1}\right)$.

Proof. It suffices to show that

$$
\int_{G} f(x) \Phi_{s}^{p,-\lambda}(x) d x=\int_{G} f(x) \Phi_{s}^{-p, \lambda}\left(x^{-1}\right) d x
$$


for all $f \in C_{c}^{\infty}(G)$. Since $\Phi_{s}^{p, \lambda}\left(k \times k^{-1}\right)=\Phi_{s}^{p, \lambda}(x), x \in G, k \in K$ and $\Phi_{s}^{p, \lambda *} \chi_{s}=$ $\Phi_{s}^{p, \lambda}$, we only need to consider those $f$ in $I_{c, s}(G)$. Thus let $f \in I_{c, s}(G)$, by (10)

$$
\begin{aligned}
\begin{aligned}
\int_{G} f(x) \Phi_{s}^{p, \lambda}(x) d x & =\int_{G} f\left(x^{-1}\right) \Phi_{s}^{p, \lambda}\left(x^{-1}\right) d x \\
& =\int_{G} f\left(x^{-1}\right) \exp (-(i \lambda+1) \rho(H(x))) D_{-p,-p}^{s}(\kappa(x)) d x \\
& =\int_{K} \int_{A_{p}} \int_{N} f\left(n^{-1} a_{t}^{-1} k^{-1}\right) e^{-(i \lambda+1) t} D_{-p,-p}^{s}(k) e^{2 t} d k d t d n \\
& =\int_{K} \int_{A_{p}} \int_{N} f\left(k n a_{t}\right) e^{(i \lambda-1) t} D_{-p,-p}^{s}\left(k^{-1}\right) d k d t d n \\
& =\int_{K} \int_{A_{p}} \int_{N} f\left(k a_{t} n\right) e^{(i \lambda+1) t} D_{-p,-p}^{s}\left(k^{-1}\right) d k d t d n \\
\int_{G} f(x) \Phi_{s}^{-p,-\lambda}\left(x^{-1}\right) d x & =\int_{G} f(x) \exp (-(-i \lambda+1) \rho(H(x))) D_{p p}^{s}(k(x)) d x \\
& =\int_{K} \int_{A_{p}} \int_{N} f\left(k a_{t} n\right) e^{(i \lambda-1) t} D_{p p}^{s}(k) e^{2 t} d k d t d n \\
& =\int_{K} \int_{A_{p}} \int_{N} f\left(k a_{t} n\right) e^{(i \lambda+1) t} D_{p p}^{s}(k) d k d t d n
\end{aligned}
\end{aligned}
$$

But $D_{-p,-p}^{s}\left(k^{-1}\right)=D_{p p}^{s}(k)$ by (6), hence the lemma.

Let $w_{1}=S_{1}^{2}+S_{2}^{2}+S_{3}^{2}-R_{1}^{2}-R_{2}^{2}-R_{3}^{2}$ and $w_{2}=R_{1} S_{1}+R_{2} S_{2}+$ $R_{3} S_{3}$. Then $\left\{w_{1}, w_{2}\right\}$ generates the center 3. It is easy to see that $S_{1}=R_{2}-N_{2}, S_{2}=N_{1}-R_{1}$ and $N_{1} R_{1}=R_{1} N_{1}-S_{3}, N_{2} R_{2}=R_{2} N_{2}-S_{3}$, substitute into $w_{1}, w_{2}$ we get

$$
\begin{gathered}
w_{1}=S_{3}^{2}+2 S_{3}-R_{3}^{2}+N_{1}^{2}+N_{2}^{2}-2\left(R_{1} N_{1}+R_{2} N_{2}\right) \\
w_{2}=R_{3} S_{3}+R_{3}-R_{1} N_{2}+R_{2} N_{1} .
\end{gathered}
$$

Use the formula for $\Phi_{s}^{p, \lambda}(x)$ in the above lemma, a direct computation gives us

$$
w_{1} \Phi_{s}^{p, \lambda}(1)=p^{2}-\lambda^{2}-1, \quad w_{2} \Phi_{s}^{p, \lambda}(1)=p \lambda .
$$

Now, $\Phi_{s}^{p, \lambda}=1 /(2 s+1)$ Trace $\left(\Psi_{s}^{p, \lambda}\right)$, and for $x \in G$, we can write $x=$ $k_{1} a_{t} k_{2}, k_{1}, k_{2} \in K, a_{t} \in A_{p}^{+}$, so $\Psi_{s}^{p, \lambda}(x)=\Psi_{s}^{p, \lambda}\left(k_{1} a_{t} k_{2}\right)=D^{s}\left(k_{1}\right) \Psi_{s}^{p, \lambda}\left(a_{t}\right) D^{s}\left(k_{2}\right)$. Then this function determined by the restriction of $\Psi_{s}^{p, \lambda}$ to $A_{p}^{+}$. Let $t \neq 0$, define $\operatorname{Ad}\left(a_{t}^{-1}\right) X=a_{t}^{-1} X a_{t}, X \in g_{0}$; then we have

$$
\begin{aligned}
& \operatorname{Ad}\left(a_{t}^{-1}\right) R_{1}=\cosh t \cdot R_{1}-\sinh t \cdot S_{2}, \\
& \operatorname{Ad}\left(a_{t}^{-1}\right) R_{2}=\cosh t \cdot R_{2}+\sinh t \cdot S_{1} .
\end{aligned}
$$

By substitution, we get 


$$
\begin{aligned}
w_{1}= & S_{3}^{2}+2 \operatorname{coth} t \cdot S_{3}+\operatorname{coth}^{2} t \cdot\left(R_{1}^{2}+R_{2}^{2}\right) \\
& +\operatorname{csch} t \cdot \operatorname{Ad}\left(a_{t}^{-1}\right)\left(R_{1}^{2}+R_{2}^{2}\right) \\
& -2 \operatorname{coth} t \operatorname{csch} t \cdot\left(\left(\operatorname{Ad}\left(a_{t}^{-1}\right) R_{1}\right) R_{2}\right. \\
& +\left(\left(\operatorname{Ad}\left(a_{t}^{-1}\right) R_{2}\right) R_{1}\right)-\left(R_{1}^{2}+R_{2}^{2}+R_{3}^{2}\right) \\
w_{2}= & S_{3} R_{3}+\operatorname{coth} t \cdot R_{3}-\operatorname{csch} t \cdot\left(\left(\operatorname{Ad}\left(a_{t}^{-1}\right) R_{1}\right) R_{2}\right. \\
& \left.-\left(\operatorname{Ad~}\left(a_{t}^{-1}\right) R_{2}\right) R_{1}\right) .
\end{aligned}
$$

Hence for $t>0$, apply $w_{1}, w_{2}$ on $\Psi_{s}^{p, \lambda}\left(a_{t}\right)$, we get

$$
\begin{aligned}
& \frac{d^{2}}{d t^{2}} \Psi_{s}^{p, \lambda}\left(a_{t}\right)+2 \operatorname{coth} t \frac{d}{d t} \Psi_{s}^{p, \lambda}\left(a_{t}\right) \\
& \quad+\left(\operatorname{coth}^{2} t-\operatorname{csch}^{2} t\right) D^{s}\left(R_{1}^{2}+R_{2}^{2}\right) \Psi_{s}^{p, \lambda}\left(a_{t}\right) \\
& \quad+\operatorname{coth} t \operatorname{csch} t\left(X \Psi_{s}^{p, \lambda}\left(a_{t}\right) Y+Y \Psi_{s}^{p, \lambda}\left(a_{t}\right) X\right) \\
& \quad+s(s+1) \Psi_{s}^{p, \lambda}\left(a_{t}\right)=\left(p^{2}-\lambda^{2}-1\right) \Psi_{s}^{p, \lambda}\left(a_{t}\right) .
\end{aligned}
$$

$$
\begin{aligned}
D^{s}( & \left.R_{3}\right) \frac{d}{d t} \Psi_{s}^{p, \lambda}\left(a_{t}\right)+\operatorname{coth} t D^{s}\left(R_{3}\right) \Psi_{s}^{p, \lambda}\left(a_{t}\right) \\
& -\frac{1}{2} \operatorname{csch} t\left(X \Psi_{s}^{p, \lambda}\left(a_{t}\right) Y-Y \Psi_{s}^{p, \lambda}\left(a_{t}\right) X\right)=p \lambda \Psi_{s}^{p, \lambda}\left(a_{t}\right)
\end{aligned}
$$

where $X=D^{s}\left(R_{1}\right)-i D^{s}\left(R_{2}\right), Y=-D^{s}\left(R_{1}\right)-i D^{s}\left(R_{2}\right)$. Since $u_{\theta} a_{t}=a_{t} u_{\theta}$, $u_{\theta} \in M, a_{t} \in A_{p}$, by (5) we see that $\Psi_{s}^{p, \lambda}\left(a_{t}\right)$ is a diagonal matrix, so let $\Psi_{s, j}^{p, \lambda}$ be the $j$ th diagonal element, $j=-s,-s+1, \cdots, s$, we see from (18) and (6)

$$
\begin{aligned}
& -i j \frac{d}{d t} \Psi_{s, j}^{p, \lambda}\left(a_{t}\right)-i j \operatorname{coth} t \Psi_{s, j}^{p, \lambda}\left(a_{t}\right) \\
& \quad-\frac{i}{2} \operatorname{csch} t\left((s-j)(s+j+1) \Psi_{s, j+1}^{p, \lambda}\left(a_{t}\right)\right. \\
& \left.\quad-(s-j)(s-j+1) \Psi_{s, j-1}^{p, \lambda}\left(a_{t}\right)\right)=p \lambda \Psi_{s, j}^{p, \lambda}\left(a_{t}\right) .
\end{aligned}
$$

Hence for $j=s, s-1, s-2, \cdots,-s+1$, we get

$$
\begin{aligned}
(s+ & j)(s-j+1) \operatorname{csch} t \Psi_{s, j-1}^{p, \lambda}\left(a_{t}\right) \\
= & 2 j \frac{d}{d t} \Psi_{s, j}^{p, \lambda}\left(a_{t}\right)+2 j \operatorname{coth} t \Psi_{s, j}^{p, \lambda}\left(a_{t}\right) \\
& \quad-2 i p \lambda \Psi_{s, j}^{p, \lambda}\left(a_{t}\right)+(s-j)(s+j+1) \operatorname{csch} t \Psi_{s, j+1}^{p, \lambda}\left(a_{t}\right) .
\end{aligned}
$$

Therefore, $\Psi_{s}^{p, \lambda}\left(a_{t}\right)$ is determined by knowing $\Psi_{s, s}^{p, \lambda}\left(a_{t}\right), t>0$. Consider the sth diagonal element of $(17)+2 i \operatorname{coth} t \cdot(18)$, we find that $\Psi_{s, s}^{p, \lambda}\left(a_{t}\right)$ satisfies the following differential equation

$$
\begin{aligned}
\varphi^{\prime \prime}(t) & +2(1+s) \operatorname{coth} t \varphi^{\prime}(t) \\
& +\left((s+1)^{2}-p^{2}+\lambda^{2}-2 i p \lambda \operatorname{coth} t\right) \varphi(t)=0 .
\end{aligned}
$$


This is a differential equations with regular singular point at $t=0$. The inditial equation $f(z)=z(z+1+s)$, so we have $z_{1}=0$ and $z_{2}=-(1+s)$ as roots for $f(z)=0$. From the general theory of such differential equation [e.g. 1, Ch. 4] we have

Proposition 3.3. Two linearly independent solutions of (21) can be represented in the following form

$$
\begin{gathered}
\varphi_{1}(t)=t^{z_{1}} U_{1}(t)=U_{1}(t) \\
\varphi_{2}(t)=t^{z_{2}} U_{2}(t)+\alpha \varphi_{1}(t) \ln t
\end{gathered}
$$

here $U_{1}$ and $U_{2}$ are analytic on $[0, \infty)$ with $U_{1}(0)=U_{2}(0)=1$ and $\alpha$ is some constant.

CoROLLARY 1. The function $\Psi_{s, s}^{p, \lambda}\left(a_{t}\right)=\varphi_{1}(t)$.

Proof. The only solutions of (21) which are bounded at $t=0$ are constant multiples of $\varphi_{1}(t)$ and we know that $\Psi_{s, s}^{p, \lambda}(1)=1$.

Let $\varphi_{1}(t)=\sum_{j=0}^{\infty} c_{j} t^{j}$. We will compute the coefficients $c_{j}$ more explicitly. Since $\lim _{t \rightarrow 0} t \operatorname{coth} t=1$, we get

$$
\cot h t=\frac{1}{t}+\sum_{j=0}^{\infty} a_{j} t^{j}
$$

with $g(t)=\sum_{j=0}^{\infty} a_{j} t^{j}$ analytic at $t=0$. Substitute $\varphi_{1}(t)$ into (21), we get

$$
2(1+s) c_{1}-2 i p \lambda c_{0}=0
$$

and the recursion formula, $j=2,3, \cdots$

$$
\begin{aligned}
j(j+1+2 s) c_{j}= & {\left[p^{2}-\lambda^{2}-(s+1)^{2}\right] c_{j-2}-2(1+s) \sum_{r=1}^{j-1} r c_{r} a_{j-1-r} } \\
& +2 i p \lambda \sum_{r=0}^{j-1} c_{r} a_{j-2-r} .
\end{aligned}
$$

COROLLARY 2. Two spherical functions $\Phi_{s}^{p_{1}, \lambda_{1}}$ and $\Phi_{s}^{p_{2}, \lambda_{2}}$ of type $s$ are equal if and only if $\left(p_{2}, \lambda_{2}\right)= \pm\left(p_{1}, \lambda_{1}\right)$ or $\left(p_{2}, \lambda_{2}\right)= \pm i\left(\lambda_{1},-p_{1}\right)$.

Proof. From earlier discussion, it suffices to consider the functions $\Psi_{s, s}^{p_{1}, \lambda_{1}}\left(a_{t}\right)$ and $\Psi_{s, s}^{p_{2}, \lambda_{2}}\left(a_{t}\right)$, hence their corresponding coefficients derived from (25) and (26). Clearly then it is equivalent to have $p_{1} \lambda_{1}=p_{2} \lambda_{2}$ and $p_{1}^{2}-\lambda_{1}^{2}=p_{2}^{2}-\lambda_{2}^{2}$ and the corollary follows.

Proposition 3.4. $\Phi_{s}^{p, \lambda}$ is bounded if $\lambda=\sigma+i b$ with $\sigma, b \in \boldsymbol{R}$ and $|b| \leqq 1$. 
Proof. Let $x \in G$ and write $x=k_{1} a_{t} k_{2}$ with $t \geqq 0$. Then

$$
\begin{aligned}
\Phi_{s}^{p, \lambda}\left(x^{-1}\right) & =\Phi_{s}^{p, \lambda}\left(\left(k_{1} a_{t} k_{2}\right)^{-1}\right)=\Phi_{s}^{p, \lambda}\left(\left(k_{2} k_{1} a_{t}\right)^{-1}\right) \\
& =\int_{K} \exp \left(-(i \lambda+1) \rho\left(H\left(a_{t} k\right)\right) D_{-p,-p}^{s}\left(k^{-1} k_{2} k_{1} k\left(a_{t} k\right)\right) d k .\right.
\end{aligned}
$$

Now, write $k=u_{\varphi_{1}} v_{\theta} u_{\varphi_{2}}$, then $a_{t} k=\left(u_{\varphi_{1}} v_{\theta}, u_{\varphi_{2}}\right) a_{t}, n, n \in N$,

$$
\begin{aligned}
& e^{t^{\prime}}=e^{t} \cos ^{2} \frac{\theta}{2}+e^{-t} \sin ^{2} \frac{\theta}{2} \\
& \cos \frac{\theta^{\prime}}{2}=e^{\left(t-t^{\prime}\right) / 2} \cos \frac{\theta}{2}, \quad \sin \frac{\theta^{\prime}}{2}=e^{-\left(t+t^{\prime}\right) / 2} \sin \frac{\theta}{2}, \quad 0 \leqq \theta^{\prime} \leqq \pi .
\end{aligned}
$$

Thus by (4) and (5) we get

$$
\Phi_{s}^{p, \lambda}\left(x^{-1}\right)=\frac{1}{2} \int_{0}^{\pi} \exp \left(-(i \lambda+1) t^{\prime}\right) D_{-p,-p}^{s}\left(v_{\theta}^{-1} k_{2} k_{1} v_{\theta^{\prime}}\right) \sin \theta d \theta .
$$

If $t=0$, then $t^{\prime}=0$ and the integral (29) bounds by 1 . If $t>0$, by (28) with change of variable gives

$$
\Phi_{s}^{p, \lambda}\left(x^{-1}\right)=\frac{1}{2 \sinh t} \sum_{j=s}^{s} \int_{-t}^{t} e^{-\lambda t^{\prime}} D_{-p, j}^{s}\left(v_{\theta}^{-1}\right) D_{j, j}^{s}\left(k_{2} k_{1}\right) D_{\jmath,-p}^{s}\left(v_{\theta^{\prime}}\right) d t^{\prime}
$$

and

$$
\left|\Phi_{s}^{p, \lambda}\left(x^{-1}\right)\right| \leqq \frac{1}{2 \sinh t} \int_{-t}^{t} e^{b t^{\prime}} d t=\frac{\sinh t}{b \sinh t} \leqq 1
$$

4. The analogue of Paley-Wiener theorem. Let

$$
B_{s}=\{(p, \lambda): p=-s,-s+1, \cdots, s ; \lambda \in C\} .
$$

For each pair $(p, \lambda) \in B_{s}$, there corresponds a spherical functions $\Phi_{s}^{p, \lambda}$ of type $s$. Let $f \in I_{c, s}(G)$, the Fourier-Laplace transform $\hat{f}$ of $f$ is a function defined on $B_{s}$ by

$$
\hat{f}(p, \lambda)=\int_{G} f(x) \Phi_{s}^{p, \lambda}(x) d x .
$$

Given $f \in I_{c}(G)$. Let $B_{f}=\left\{a_{t} \in A_{p}: f\left(k a_{t}\right) \neq 0\right.$ for some $\left.k \in K\right\}$. We say that $f$ has support in the ball of radius $R$ if $\sup \left\{|t|: a_{t} \in\right.$ $\left.B_{f}\right\} \leqq R$. Clearly $f$ has compact support if and only if there exists an $R$ which is finite. For each $D^{s} \in \hat{K}$, define

$$
F_{f}^{s}\left(a_{t}\right)=e^{t} \int_{K} \int_{N} f\left(k a_{t} n\right) D^{s}\left(k^{-1}\right) d k d n .
$$

This gives a map of $A_{p}$ to the space of linear operators $L\left(E_{8}\right)$ on $E_{8}$. It is easy to see that $F_{f}^{s}=F_{f_{s}}^{s}, f \in I_{c}(G)$ and $f_{s}=f * \chi_{s}$. 
LEMMA 4.1. Let $n \in N, a_{t} \in A_{p}$ and write $a_{t} n=k_{1} a_{t_{1}} k_{2}$ for some $k_{1}, k_{2} \in K$. Then $\left|t_{1}\right| \geqq|t|$.

Proof. Let $n=\left(\begin{array}{ll}1 & z \\ 0 & 1\end{array}\right), z \in C$ and $k_{j}=\left(\begin{array}{cc}\alpha_{j} & \beta_{j} \\ \bar{\beta}_{j} & \bar{\alpha}_{j}\end{array}\right)$ with $\left|\alpha_{j}\right|^{2}+$ $\left|\beta_{j}\right|^{2}=1, j=1,2$. Equating the corresponding matrix coefficients from $a_{t} n k_{2}^{-1}$ yields $e^{t}+\left(1+|z|^{2}\right) e^{-t}=e^{t_{1}}+e^{-t_{1}}$, i.e., $2 \cosh t_{1}+e^{t}|z|^{2}$. Thus $\left|t_{1}\right| \geqq|t|$.

Proposition 4.2. Let $f \in I_{c, s}(G)$ have support in the ball of radius $R$, then $F_{f}^{s}$ is $C^{\infty}$ with support in $\left\{a_{t}:|t| \leqq R\right\}$.

Proof. Suppose $F_{f}^{s}\left(a_{t}\right) \neq 0$, then $f\left(k a_{t} n\right) \neq 0$ for some $k \in K$, $n \in N$. Now $k a_{t} n=k_{1} a_{t_{1}} k_{2}$ for some $k_{1}, k_{2} \in K$ and $a_{t_{1}} \in A_{p}$. Thus $a_{t} n=k^{-1} k_{1} a_{t_{1}} k_{2}$ and $f\left(k_{2} k_{1} a_{t_{1}}\right)=f\left(k_{1} a_{t_{1}} k_{2}\right)=f\left(k a_{t} n\right) \neq 0$. By the above lemma and the assumption we get $|t| \leqq\left|t_{1}\right| \leqq R$. Differentiability is clear.

Proposition 4.3. The $\operatorname{map} f \rightarrow F_{f}^{s}$ is a one-to-one algebra homomorphism of $I_{c, s}(G)$ into $C_{c}^{\infty}\left(A_{p}, L\left(E_{s}\right)\right)$.

Proof. Let $f, g \in I_{c, s}(t)$, use Fubini's theorem repeatedly

$$
\begin{aligned}
F_{f^{*} g}^{s}\left(a_{t}\right)= & e^{t} \int_{K} \int_{N}(f * g)\left(k a_{t} n\right) D^{s}\left(k^{-1}\right) d k d n \\
= & e^{t} \int_{K} \int_{N} \int_{G} f\left(k a_{t} n x^{-1}\right) g(x) D^{s}\left(k^{-1}\right) d x d k d n \\
= & e^{t} \int_{K} \int_{N} \int_{K} \int_{A_{p}} \int_{N} f\left(k a_{t} n n_{1}^{-1} a_{t_{1}}^{-1} k_{1}^{-1}\right) g\left(k_{1} a_{t_{1}} n_{1}\right) \\
& \times e^{2 t_{1}} D^{s}\left(k^{-1}\right) d k_{1} d t_{1} d n_{1} d k d n \\
= & \int_{A_{p}} \int_{K} \int_{N} \int_{K} \int_{N} f\left(k a_{t} a_{t_{1}}^{-1} n\right) g\left(k_{1} a_{t_{1}} n_{1}\right) e^{t_{1}} \cdot e^{t-t_{1}} D^{s}\left(k^{-1}\right) \\
& \times D^{s}\left(k_{1}^{-1}\right) d k_{1} d n_{1} d k d r d t_{1} \\
= & \int_{A_{p}} F_{f}^{s}\left(a_{t} a_{t_{1}}^{-1}\right) F_{g}^{s}\left(a_{t_{1}}\right) d t_{1}=F_{f}^{s} * F_{g}^{s}\left(a_{t}\right) .
\end{aligned}
$$

The linearity is trivial, hence it is algebra homomorphism. As for one-to-one, given $f \in I_{c, s}(G)$ and $F_{f}^{s} \equiv 0$, to show $f \equiv 0$. Note first that $F_{f}^{s}\left(a_{t}\right) D^{s}\left(u_{\theta}\right)=D^{s}\left(u_{\theta}\right) F_{f}^{s}\left(a_{t}\right)$, hence $F_{f}^{s}\left(a_{t}\right)$ is a diagonal matrix. From (10) and Lemma 3.2, we see that if $F_{f, p}^{s}\left(a_{t}\right)$ is the pth diagonal element of $F_{f}^{s}\left(a_{t}\right)$,

$$
\int_{A_{p}} F_{f, p}^{s}\left(a_{t}\right) e^{-i \lambda t} d t=\int_{G} f(x) \Phi_{s}^{p, \lambda}(x) d x .
$$


If $F_{f}^{s} \equiv 0$, then $F_{f, p}^{s} \equiv 0$ for all $p$, hence $\hat{f}(p, \lambda)=0$ for all $p, \lambda$. Thus $U^{p, \lambda}(f)=0$ for all $p, \lambda$. But the set $\left\{U^{p, \lambda}\right\}$ forms a complete set of representations on $G$, thus we get $f=0$.

\section{CoRollaRY. $I_{c, s}(G)$ is commutative.}

For each nonnegative real number $R$, let $H_{s}(R)$ be the set of functions $g$ defined on $B_{s}$ satisfying (i) $g$ is entire holomorphic in $\lambda$; (ii) $g(p, \lambda)=g(-p,-\lambda),(p, \lambda) \in B_{s}$; (iii) $g(p, \lambda)=g(i \lambda,-i p)$ if both $(p, \lambda)$ and $(i \lambda,-i p)$ are in $B_{s}$; (iv) given a positive integer $m$, there exists a constant $C_{m}$ such that $|g(p, \lambda)| \leqq C_{m}(1+|\lambda|)^{-m} \exp R|\eta|$, $\lambda=\xi+i \eta \in \boldsymbol{R}+i \boldsymbol{R}$. Let $H_{s}$ be the union of all the $H_{s}(R)$.

Given $f$ in $I_{c, s}(G)$, by Corollary 2 of Proposition 3.3 we see the function $\hat{f}$ defined in (30) satisfies conditions (i), (ii), (iii) of the definition of $H_{s}$. By (32), $\hat{f}(p, \lambda)$ is just the usual Fourier transform of the function $F_{f, p}^{s}$ on the real line, which is $C^{\infty}$ with compact support, hence $\hat{f}$ is holomorphic in $\lambda$. If $f$ has support in the ball of radius $R$, so is $F_{f}^{s}$, hence the classical Paley-Wiener theorem asserts that $\hat{f} \in H_{s}(R)$. Thus we have a linear map $f \rightarrow \hat{f}$ of $I_{c, s}(G)$ into $H_{s}$ such that if $f$ has support in the ball of radius $R$, we get $\hat{f} \in H_{s}(R)$. We want to show that this map is also onto now.

In the inversion formula (8), when $f \in I_{c, s}(G)$, it is easy to see that Trace $\left(U^{p, \lambda}\left(x^{-1}\right) U^{p, \lambda}(f)\right)=(2 s+1) \hat{f}(p, \lambda) \Phi_{s}^{p, \lambda}\left(x^{-1}\right)$ for $p=-s,-s+$ $1, \cdots, s$; and $U^{p, \lambda}(f)=0$ otherwise. Thus we have

$$
f(x)=\frac{2 s+1}{4 \pi^{3}} \sum_{p=-s}^{s}\left(p^{2}+\lambda^{2}\right) \hat{f}(p, \lambda) \Phi_{s}^{p, \lambda}\left(x^{-1}\right) d \lambda .
$$

Lemma 4.4. Let $g \in H_{s}(R)$ and define

$$
f_{1}(x)=\sum_{p=-s}^{s} \int_{\lambda=-\infty}^{\infty}\left(p^{2}+\lambda^{2}\right) g(p, \lambda) \Phi_{s}^{p, \lambda}\left(x^{-1}\right) d \lambda .
$$

Then $f_{1} \in I_{c, s}(G)$ and $f_{1}$ has support in the ball of radius $R$.

Proof. Since $g(p, \lambda)$ decreases rapidly at infinity on $\lambda$ and $\Phi_{s}^{p, \lambda}$ is $C^{\infty}$ and bounded when $\lambda$ is real, the integral converges absolutely and defines a $C^{\infty}$ function on $G$. By the property of $\Phi_{s}^{p, \lambda}$, it is clear that $f_{1}\left(k \times k^{-1}\right)=f_{1}(x), k \in K, x \in G$ and $f_{1} * \chi_{s}=f_{1}$. It remains to show that $f_{1}$ has support in the ball of radius $R$. Thus let $x=k_{1} a_{t}$ with $k_{1} \in K$ and $t \neq 0$. Since $a_{t} \in B_{f_{1}}$ if and only if $a_{-t} \in B_{f_{1}}$, may assume that $t>0$. Using the expression and notation in Proposition 3.4, we get

$$
\begin{aligned}
f_{1}\left(k_{1} a_{t}\right)= & \frac{1}{2 \sinh t} \sum_{p, j=-s}^{s} D_{j, j}^{s}\left(k_{1}\right) \int_{\lambda=-\infty}^{\infty} \int_{t^{\prime}=-t}^{t}\left(p^{2}+\lambda^{2}\right) g(p, \lambda) e^{-i \lambda t^{\prime}} \\
& \times D_{-p, j}^{s}\left(v_{\theta}^{-1}\right) D_{j,-p}^{s}\left(v_{\theta^{\prime}}\right) d t^{\prime} d \lambda .
\end{aligned}
$$


For each $p, j=-s,-s+1, \cdots, s$, define

(36) $f_{p, j}(t)=\int_{\lambda=-\infty}^{\infty} \int_{t^{\prime}=t}^{t}\left(p^{2}+\lambda^{2}\right) g(p, \lambda) e^{-i \lambda t^{\prime}} D_{-p, j}^{s}\left(v_{\theta}^{-1}\right) D_{j,-p}^{s}\left(v_{\theta^{\prime}}\right) d t^{\prime} d \lambda$.

Let $t>R$, to show $f_{1}\left(k_{1} a_{t}\right)=0$, it suffices to show that $\sum_{p=-s}^{s} f_{p, j}(t)=0$ for all $j$. Let

$$
h_{p}\left(t^{\prime}\right)=\int_{-\infty}^{\infty}\left(p^{2}+\lambda^{2}\right) g(p, \lambda) e^{-i \lambda t^{\prime}} d \lambda .
$$

By the classical Paley-Wiener theorem, $h_{p}\left(t^{\prime}\right)=0$ if $t^{\prime}>R$. Thus

$$
f_{p, j}(t)=\int_{-\infty}^{\infty} h_{p}\left(t^{\prime}\right) D_{-p, j}^{s}\left(v_{\theta}^{-1}\right) D_{j,-p}^{s}\left(v_{\theta^{\prime}}\right) d t^{\prime} .
$$

Put $x_{1}=e^{t^{\prime}}, x_{2}=e^{-t^{\prime}}$, then by (5), (28) we get

$$
\begin{aligned}
D_{-\cdot p, j}^{s}\left(v_{\theta}^{-1}\right) D_{j,-p}^{s}\left(v_{\theta^{\prime}}\right)= & \frac{(-1)^{s+j} e^{-j t}}{(s+j) !(s-j) !(2 \sin h t)^{2 s}} e^{-p t^{\prime}} \frac{\partial^{2 s}}{\partial x_{1}^{s-p} \partial x_{2}^{s+p}} \\
& \times\left[\left(x_{1} x_{2}-e^{-t}\left(x_{1}+x_{2}\right)+e^{-2 t}\right)^{s-j}\right. \\
& \left.\times\left(x_{1} x_{2}-e^{t}\left(x_{1}+x_{2}\right)+e^{2 t}\right)^{s+j}\right] .
\end{aligned}
$$

The above expression is just the linear combination of terms

$$
e^{-p t^{\prime}} \frac{\partial^{2 s}}{\partial x_{1}^{s-p} \partial x_{2}^{s+p}}\left[\left(x_{1} x_{2}\right)^{r_{1}}\left(x_{1}^{r_{2}}+x_{2}^{r_{i}}\right)\right]
$$

with coefficients as functions of $t$, and $r_{1}, r_{2} \geqq 0, r_{1}+r_{2} \leqq 2 s$. Pick one of these terms and consider the two integrals

$$
\begin{aligned}
& \sum_{p=-s}^{s} \int_{-\infty}^{\infty} h_{p}\left(t^{\prime}\right) e^{-p t^{\prime}} \frac{\partial^{2 s}}{\partial x_{1}^{s-p} \partial x_{2}^{s+p}}\left[x_{1}^{r_{1}+r_{2}} x_{2}^{r_{1}}\right] d t^{\prime} \\
& =\sum_{p=\max \left\{-s, s-r_{1}-r_{2}\right\}}^{\min \left\{s, r_{1}-s\right\}} \int_{-\infty}^{\infty} h_{p}\left(t^{\prime}\right) \frac{\left(r_{1}+r_{2}\right) ! r_{1} !}{\left(r_{1}+r_{2}-s+p\right) !\left(r_{1}-s-p\right) !} e^{\left(r_{2}+p\right) t^{\prime}} d t^{\prime} \\
& =2 \pi \sum_{p=s-r_{1}-r_{2}}^{r_{1}-s} \frac{\left(r_{1}+r_{2}\right) ! r_{1} !}{\left(r_{1}+r_{2}-s+p\right) !\left(r_{1}-s-p\right) !} \\
& \times\left[p^{2}+\left(-i\left(r_{2}+p\right)\right)^{2}\right] g\left(p,-i\left(r_{2}+p\right)\right) \\
& =-2 \pi \sum_{p=s-r_{1}-r_{2}}^{r_{1}-s} \frac{\left(r_{1}+r_{2}\right) ! r_{1} !}{\left(r_{1}+r_{2}-s+p\right) !\left(r_{1}-s-p\right) !} \\
& \times r_{2}\left(r_{2}+2 p\right) g\left(p,-i\left(r_{2}+p\right)\right) \\
& \sum_{p=-s}^{s} \int_{-\infty}^{\infty} h_{p}\left(t^{\prime}\right) e^{-p t^{\prime}} \frac{\partial^{2 s}}{\partial x_{1}^{s-p} \partial x_{2}^{s+p}}\left[x_{1}^{r_{1}} x_{2}^{r_{1}+r_{2}}\right] d t^{\prime} \\
& =2 \pi \sum_{p=\max \left\{-s, s-r_{1}\right\}}^{\min \left\{s, r_{1}+r_{2}-s\right\}} \frac{\left(r_{1}+r_{2}\right) ! r_{1} !}{\left(r_{1}-s+p\right) !\left(r_{1}+r_{2}-s-p\right) !} \int_{-\infty}^{\infty} h_{p}\left(t^{\prime}\right) e^{\left(p-r_{2}\right) t} d t^{\prime} \\
& =2 \pi \sum_{p=s-r_{1}}^{r_{1}+r_{2}-s} \frac{r_{1} !\left(r_{1}+r_{2}\right) !}{\left(r_{1}-s+p\right) !\left(r_{1}+r_{2}-s-p\right) !} r_{2}\left(2 p-r_{2}\right) g\left(p, i\left(r_{2}-p\right)\right) \text {. }
\end{aligned}
$$


By changing the index and the fact that

$$
g\left(p, i\left(r_{2}-p\right)\right)=g\left(p-r_{2},-i p\right),
$$

we get the sum of (40) and (41) is zero. Now the lemma is clear.

Combine the above discussion, we get the following analogue of Paley-Wiener theorem.

Proposition 4.5. The Fourier transform $f$ to $\hat{f}$ defined in (30) is a one-to-one algebra homomorphism of $I_{c, s}(G)$ onto $H_{s}$. A function $f$ in $I_{c, s}(G)$ has support in the ball of radius $R$ if and only if $\hat{f}$ is in $H_{s}(R)$.

Let $L_{s}^{1}(G)$ be the closure of $I_{c, s}(G)$ in $L^{\prime}(G)$. Given $f \in L_{s}^{1}(G)$, by Proposition 3.4, the integral

$$
\hat{f}(p, \lambda)=\int_{G} f(x) \Phi_{s}^{p, \lambda}(x) d x
$$

is defined for $(p, \lambda) \in B_{s}$ with $\lambda=\xi+i \eta,|\eta| \leqq 1$. Then we have the following analogue of Riemann Lebesgue lemma.

Proposition 4.6. Let $f \in L_{s}^{1}(G)$ and define $\hat{f}$ as in (42), then $\lim _{\xi \rightarrow \pm \infty} \hat{f}(p, \xi+i \eta)=0$ uniformly for $|\eta| \leqq 1$.

Proof. Given $\varepsilon>0$, choose $g$ in $I_{c, s}(G)$ such that $\|f-g\|_{1}<\varepsilon / 2$. But then we have

$$
|\hat{f}(p, \lambda)-\hat{g}(p, \lambda)| \leqq \int_{G}|f(x)-g(x)| d x<\varepsilon / 2 .
$$

Choose $R, C$ such that

$$
|\widehat{g}(p, \lambda)| \leqq C(1+|\lambda|)^{-1} \exp R|\eta| \leqq C(1+|\lambda|)^{-1} \exp R
$$

since $|\eta| \leqq 1$. Combine (43), (44) we get $|\hat{f}(p, \lambda)|<\varepsilon$ when $|\xi|$ is large enough.

Let $B=\{(s, p, \lambda): s$ is a nonnegative integer or half integer, $\left.(p, \lambda) \in B_{s}\right\}$. Given $f \in I_{c}(G)$ and $(s, p, \lambda) \in B$, define

$$
\hat{f}(s, p, \lambda)=\int_{G} f(x) \Phi_{s}^{p, \lambda}(x) d x .
$$

It is clear that $\hat{f}(s, p, \lambda)=\hat{f}_{s}(p, \lambda)$.

Lemma 4.7. Let $f \in I_{c}(G)$. Then $f$ has support in the ball of radius $R$ if and only if $f_{s}$ has support in the ball of radius $R$ for all s. 
Proof. By definition, $f_{s}(x)=\int_{K} f\left(k^{-1} x\right) \chi_{s}(k) d k$. Thus if $f$ has support in the ball of radius $R$ and $f_{s}\left(k_{1} a_{t}\right) \neq 0$ with $k_{1} \in K, a_{t} \in A_{p}$, we have $f\left(k^{-1} k_{1} a_{t}\right) \neq 0$ for some $k \in K$ and therefore $|t| \leqq R$. The converse follows from the fact that $\sum_{s} f_{s}$ converges to $f$ absolutely, [8, vol. I, p. 264].

Proposition 4.8. The map $f \rightarrow \hat{f}$ defined in (45) is a one-to-one algebra homomorphism of $I_{c}(G)$ into the algebra of all functions $g$ on $B$ satisfying (i) $g(s, p, \lambda)$ is entire holomorphic in $\lambda$, (ii) $g(s, p, \lambda)=$ $g(s,-p,-\lambda),(s, p, \lambda) \in B$, (iii) $g(s, p, \lambda)=g(s, i \lambda,-i p)$ if both $(s, p, \lambda)$ and $(s, i \lambda,-i p)$ are in $B$, (iv) there exists $R>0$, for each given positive integer $m$, there exists $C_{m, s}$ such that

$$
|g(s, p, \lambda)| \leqq C_{m, s}(1+|\lambda|)^{-m} \exp R|\eta|, \xi+i \eta \in \boldsymbol{R}+i \boldsymbol{R} .
$$

Proof. This is clear by Proposition 4.6 and Lemma 4.7.

CoRollary. Let $f \in L^{1}(G)$. Then $\hat{f}(s, p, \lambda)$ is defined for $\lambda=\xi+$ $i \eta,|\eta| \leqq 1$ and $\lim _{\xi \rightarrow \pm \infty} \hat{f}(s, p, \xi+i \eta)=0$ for $|\eta| \leqq 1$.

\section{REFERENCES}

1. E. A. Coddington and N. Levinson, Theory of Ordinary Differential Equations, McGraw-Hill, 1955.

2. L. Ehrenpreis and F. I. Mautner, Some properties of the Fourier transform on semisimple Lie groups, I, Ann. of Math., 61 (1955), 406-439; II, III, Trans. Amer. Math. Soc., 84 (1957), 1-55; 90 (1959), 431-484.

3. R. Gangolli, On the Plancherel formula and the Paley-Wiener theorem for spherical functions on semisimple Lie groups, Ann. of Math., 93 (1971), 150-165.

4. R. Godement, $A$ theory of spherical functions I, Trans. Amer. Math. Soc., 73 (1952), 496-556.

5. S. Helgason, An analogue of the Paley-Wiener theorem for the Fourier transform on certain symmetric spaces, Math. Ann., 165 (1966), 297-308.

6. E. Hewitt and K. Ross, Abstract Harmonic Analysis, vol. II, Springer-Verlag, 1970.

7. Y. Shimizu, An analogue of the Paley-Wiener theorem for certain function spaces on the generalized Lorentz group, J. Fac. of Sci. Univ. of Tokyo, 16 (1969), 291-311. 8. G. Warner, Harmonic Analysis on Semisimple Lie Groups, Vol. I, II, SpringerVerlag, 1972.

Received June 21, 1973. This paper is based on the author's doctoral dissertation under the direction of Professor, R. Gangolli at the University of Washington. The author wishes to express his sincere thanks to Professor Gangolli for his patient and persistent advice.

UNIVERSITY OF COLORADO 



\section{PACIFIC JOURNAL OF MATHEMATICS}

EDITORS

RICHARD ARens (Managing Editor)

University of California

Los Angeles, California 90024

\section{R. A. Beaumont}

University of Washington

Seattle. Washington 98105

\section{J. DugundjI}

Department of Mathematics University of Southern California Los Angeles, California 90007

D. Gilbarg and J. Milgram

Stanford University

Stanford. California 94305

\section{ASSOCIATE EDITORS}
E. F. BECKENBACH
B. H. NEUMANN
F. WOLF
K. Yoshida

\section{SUPPORTING INSTITUTIONS}

\author{
UNIVERSITY OF BRITISH COLUMBIA \\ CALIFORNIA INSTITUTE OF TECHNOLOGY \\ UNIVERSITY OF CALIFORNIA \\ MONTANA STATE UNIVERSITY \\ UNIVERSITY OF NEVADA \\ NEW MEXICO STATE UNIVERSITY \\ OREGON STATE UNIVERSITY \\ UNIVERSITY OF OREGON \\ OSAKA UNIVERSITY
}

\author{
UNIVERSITY OF SOUTHERN CALIFORNIA \\ STANFORD UNIVERSITY \\ UNIVERSITY OF TOKYO \\ UNIVERSITY OF UTAH \\ WASHINGTON STATE UNIVERSITY \\ UNIVERSITY OF WASHINGTON

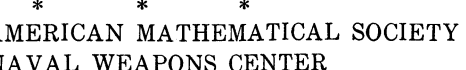

The Supporting Institutions listed above contribute to the cost of publication of this Journal, but they are not owners or publishers and have no responsibility for its content or policies.

Mathematical papers intended for publication in the Pacific Journal of Mathematics should be in typed form or offset-reproduced, (not dittoed), double spaced with large margins. Underline Greek letters in red, German in green, and script in blue. The first paragraph or two must be capable of being used separately as a synopsis of the entire paper. Items of the bibliography should not be cited there unless absolutely necessary, in which case they must be identified by author and Journal, rather than by item number. Manuscripts, in duplicate if possible, may be sent to any one of the four editors. Please classify according to the scheme of Math. Rev. Index to Vol. 39. All other communications to the editors should be addressed to the managing editor, or Elaine Barth, University of California, Los Angeles, California, 90024.

100 reprints are provided free for each article, only if page charges have been substantially paid Additional copies may be obtained at cost in multiples of 50 .

The Pacific of Journal Mathematics is issued monthly as of January 1966. Regular subscription rate: $\$ 72.00$ a year (6 Vols., 12 issues). Special rate: $\$ 36.00$ a year to individual members of supporting institutions.

Subscriptions, orders for back numbers, and changes of address should be sent to Pacific Journal of Mathematics, 103 Highland Boulevard, Berkeley, California, 94708.

\section{PUBLISHED BY PACIFIC JOURNAL OF MATHEMATICS, A NON-PROFIT CORPORATION}

Printed at Kokusai Bunken Insatsusha (International Academic Printing Co., Ltd.), 270, 3-chome Totsuka-cho. Shinjuku-ku, Tokyo 160. Japan.

Copyright (C) 1973 by Pacific Journal of Mathematics Manufactured and first issued in Japan 


\section{Pacific Journal of Mathematics}

\section{Vol. 52, No. $2 \quad$ February, 1974}

Harm Bart, Spectral properties of locally holomorphic vector-valued functions .....

J. Adrian (John) Bondy and Robert Louis Hemminger, Reconstructing infinite

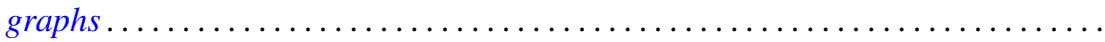

Bryan Edmund Cain and Richard J. Tondra, Biholomorphic approximation of planar

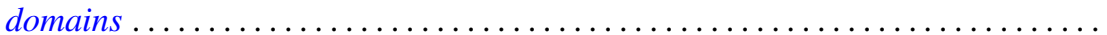

Richard Carey and Joel David Pincus, Eigenvalues of seminormal operators,

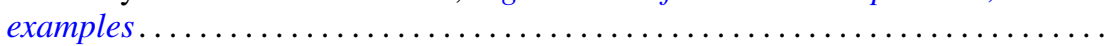

Tyrone Duncan, Absolute continuity for abstract Wiener spaces . . . . . . . . . . . . Joe Wayne Fisher and Louis Halle Rowen, An embedding of semiprime

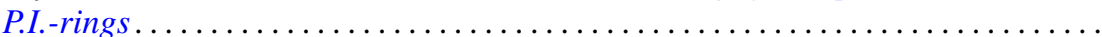

Andrew S. Geue, Precompact and collectively semi-precompact sets of semi-precompact continuous linear operators. . . . . . . . . . . . . . . .

Charles Lemuel Hagopian, Locally homeomorphic $\lambda$ connected plane continua ..... . Darald Joe Hartfiel, A study of convex sets of stochastic matrices induced by

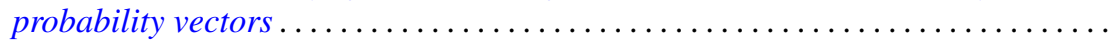

Yasunori Ishibashi, Some remarks on high order derivations $\ldots \ldots \ldots \ldots \ldots \ldots \ldots$ Donald Gordon James, Orthogonal groups of dyadic unimodular quadratic forms.

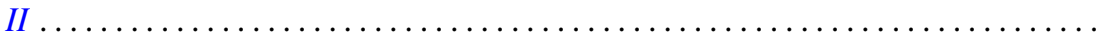

Geoffrey Thomas Jones, Projective pseudo-complemented semilattices . . . . . . . . . Darrell Conley Kent, Kelly Denis McKennon, G. Richardson and M. Schroder,

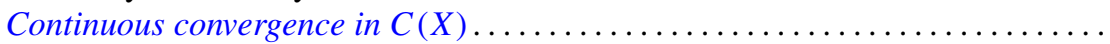

J. J. Koliha, Some convergence theorems in Banach algebras ...

Tsang Hai Kuo, Projections in the spaces of bounded linear oper

George Berry Leeman, Jr., A local estimate for typically real functions . .

475

Andrew Guy Markoe, A characterization of normal analytic spaces by the

homological codimension of the structure sheaf .........

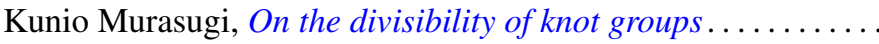

John Phillips, Perturbations of type I von Neumann algebras.

Billy E. Rhoades, Commutants of some quasi-Hausdorff matrices . .

David W. Roeder, Category theory applied to Pontryagin duality

Maxwell Alexander Rosenlicht, The nonminimality of the differential closure .

Peter Michael Rosenthal, On an inversion theorem for the general Mehler-Fock transform pair.

Alan Saleski, Stopping times for Bernoulli automorphisms

John Herman Scheuneman, Fundamental groups of compact complete locally affine complex surfaces. II. ........................

Vashishtha Narayan Singh, Reproducing kernels and operators with a cyclic vector. I. .

Peggy Strait, On the maximum and minimum of partial sums of random variables.

J. L. Brenner, Maximal ideals in the near ring of polynomials modulo 2 .

Ernst Gabor Straus, Remark on the preceding paper: "Ideals in near rings of polynomials over a field" ..........................

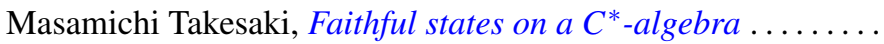

R. Michael Tanner, Some content maximizing properties of the regular simplex.

Andrew Bao-hwa Wang, An analogue of the Paley-Wiener theorem for certain

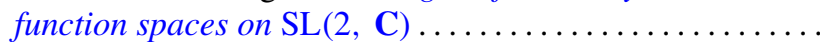

